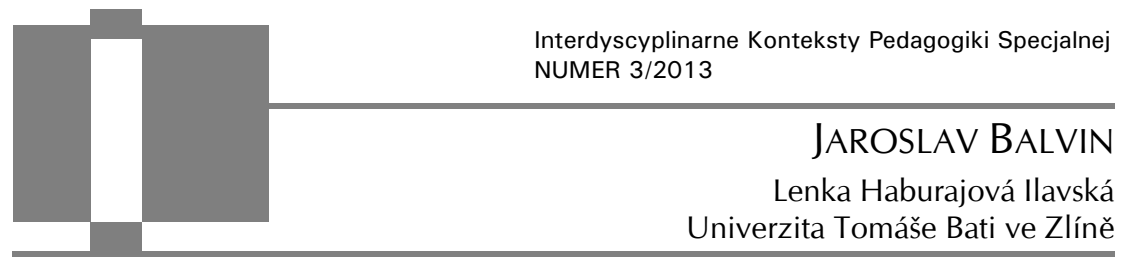

\title{
Erziehung der Roma Schüler: Möglichkeiten der Nutzung fiktiver Theorien in deren Kultur für die Erziehung in der realen Welt
}

\begin{abstract}
Balvin Jaroslav, The development of Roma pupils: The possibilities of using the case methods in cultural education [Rozwój uczniów romskich: możliwości wykorzystania metod sytuacyjnych w edukacji kulturowej]. Interdyscyplinarne Konteksty Pedagogiki Specjalnej, nr 3, Poznań 2013. Pp. 135-148. Adam Mickiewicz University Press. ISBN 978-83-232-2721-2. ISSN 2300-391X.

Thick description, thin description. Cultural way of understanding disability The aim of this article is to present disability from the cultural point of view. Special attention will be paid to Clifford Geertz and his thick description. The phrase thick description is borrowed from Gilbert Ryle. In the classic example one boy's eye involuntarily twitches while at the same time another boy winks. The physical phenomena are the same but the meaning is different. In understanding disability we have to take into consideration not only the medical, social or biopsychosocial model but cultural context as well.
\end{abstract}

KEY WORDS: education, Roma culture, methods of situational

\section{Vorwort}

Die Situations- Methoden können in der Pädagogik zu einem wichtigen Mittel werden, mit dem ein perspektivisch denkender Mensch beeinflusst werden kann. Die Nutzung dieser Methoden ist 
darauf ausgerichtet, dass der Lehrer bestimmte pädagogische Situationen schafft, zusammen mit dem Schüler versucht, diese zu bewältigen und ihn darauf vorbereitet, wie man sich in beabsichtigten und unbeabsichtigten Situationen entscheidet. Der Lehrer kann hier Methoden anwenden, welche die Fiktion als ein Mittel zur Vorbereitung der Schüler auf das wirkliche Leben nutzen. Das Wechselspiel zwischen Fiktion und Wirklichkeit (Realität) ist nach wie vor sehr wichtig beim weltanschaulichen, philosophischen und ethischen Herangehen der Roma an die Probleme des realen Lebens. Dies spiegelt sich auch in der Schaffensweise ihrer Dichter, Schriftsteller, Künstler und Schauspieler wieder. Dem Lehrer sollte die Kultur der Roma bekannt sein, die Art und Weise, wie sie an die Lösung realer Situationen herangehen. Mit der Tatsache, dass er seine Roma- Schüler lehren wird, mit Hilfe der Fiktion reale Situationen bewältigen zu können, verbindet er die multikulturelle Erziehung und Vorbereitung auf das wirkliche Leben.

\section{Erziehung und Bildung als ein Model für das reale Leben}

Erziehung und Bildung mit Hilfe von Erschaffung und Lösung pädagogischer Situationen entspricht im Wesentlichen dem realen Lebensprozess. Es handelt sich dabei immer um ein Model des realen Lebens, welches man in unterschiedlichen Situationen, sozusagen bildlich, aus dem Unterbewusstsein heraus, erleben kann. Dies schafft auch Raum für die Ausnutzung der Fiktion als einer Methode zur Entmaterialisierung, zur Abstrahierung der Realität, aber auch als einer Methode, die der Lehrer zur Vorbereitung auf das Leben in der realen Welt nutzen kann.

Diese Tatsache verbindet Pädagogik mit der Nutzung des Spiels, als ein Mittel zur Persönlichkeitsentwicklung. Das Spiel an sich fördert nicht nur den Verstand, sondern auch die sinnliche Wahrnehmung, künstlerische und gestalterische Fähigkeiten sowie die Fantasie. Zu den wichtigsten Eigenschaften eines Spiels zählt Wahl- und Entscheidungsfreiheit. Es geht hierbei um die Entwick- 
lung optimaler Entscheidungsfähigkeiten und zwar nicht nur im rationalen sondern auch im ästhetischen und ethischen Sinne. Die Fähigkeit, aus mehreren Alternativen wählen zu können, zeugt von Autonomie und moralischer Identität. Deswegen kann man beim Lösen jedes menschlichen Problems als auch in jedem Spiel eine ethische Seite finden. Da gerade der Bereich der Ethik sich mit den inneren, tiefgründigen Seiten der menschlichen Seele beschäftigt, steht diese der fiktiven Welt am nächsten. Es geht vor allem um das Herausfinden und Ausnutzen eines bestimmten "Mechanismus", welchen ein Lehrer in der Erziehung, als ein Mittel zur Vorbereitung auf die reale Welt, einsetzen kann.

\section{Das Verhältnis zwischen fiktiven Welt und Realität und deren möglicher Einsatz in der Erziehung der Schüler}

Das Spiel kann den Menschen sowohl real als auch moralisch beeinflussen. Damit der Erzieher die ethischen Situation- Spiele in der Praxis erfolgreich einsetzen kann, sollte er den "Mechanismus" kennen, welcher es möglich macht, mit Hilfe bestimmter Spielformen und fiktiver Situationen, den Menschen auf das Lösen realer Probleme vorzubereiten und ihm hilft, mit der Realität in den sich andauernd ändernden Verhältnissen sowie aus ethischer Sicht, fertig zu werden. Dieser Mechanismus hat seinen Ursprung in der menschlichen Psyche (vergl. Hlavsa, Langová, Všetečka, S. 170). Jede Situation kommt in drei Formen vor:

1. Authentische Form - unser tägliches Dasein und die Aktivitäten bei unterschiedlichen Ereignissen im Leben - REAL.

2. Bildliche Form - Imitation einer realen Situation in unterschiedlichsten Darstellungen, unter Einsatz vom vorgegebenen Zeichen- und Symbolsystem (wissenschaftliche Abstraktion ähnlich dem Spiel) - BILD.

3. Präsentierte - fiktive Form - Darstellung oder Durchleben bestimmter Situationen in der Fantasie - Träume, die imaginäre Form - IDEAL. 
Es gibt viel mehr imaginäre und bildliche Situationen als authentische. Im Gegensatz zu den authentischen Situationen, in denen grundsätzlich nur eine Möglichkeit verwirklicht wird, kann man sich von den imaginären Situationen eine größere Anzahl vorstellen. Der Mensch ist im Stande, sich seine eigene Situation und die von anderen Personen auszumalen. In der imaginären, fiktiven Form kann er diese Situation dann ausprobieren, beliebig lenken oder sich daran aktiv beteiligen. Eine besondere Rolle spielen hierbei auch Träume, mit einem großen Anteil an Weisheit oder als Projektion der menschlichen Möglichkeiten. Träume, Fiktion, Fantasie und Imagination gehören alle zu den Grundsätzen kultivierter Orientierung unseres praktischen Tun (Pešková, Schücková, 1991, S. 147-151).

Im Leben eines Menschen, in seinem praktischen Wirken wechseln sich alle drei Formen ständig $a b$, je nachdem welche Situation er augenblicklich lösen muss, was auf seinem Tagesplan steht. Und gerade diese Tatsache nutzen wir auch zur moralischer Kultivierung des menschlichen Tun, mit Hilfe - einer der alternativen Erziehungsmethoden - der ethischen Situationsspiele. Ich meine, dass diese Methode zur Vorbereitung eines Menschen auf die Lösung verschiedener Lebenssituationen genutzt werden kann und bereichert diesen Prozess um die Größe der ethischen Werte.

\section{Fiktion, Spiel, Situation: Möglichkeiten der Persönlichkeitskultivierung eines Roma- Schülers}

Mit Hilfe von Spielen, kommunikativen Methoden, Einsatz von Drama- Elementen sowie ethischen Situationsspielen (aktivierenden Spielen) kann sich der Schüler zu einer autonomen Person entwickeln. Nimmt er an einem Rollenspiel teil, kann er auf der eigenen Haut, jedoch unter vorgegebenen Model- Bedingungen erleben, wie stark die Macht der Urteilsgebung (Beurteilung) ist und die Haltung beim Lösen und Durchleben der Situationen aus des Schulleben ausschaut. Ich gehe hierbei von einem langzeitigen 
Programm aus, welches sich mit der Entwicklung multikultureller Beziehungen zwischen der Majorität (Allgemeinheit) und der Roma- Minorität (Roma- Minderheit) beschäftigt. Man muss hier quasi "von unten“ anfangen, den Roma- Schüler als den Ausgangspunkt für den bevorstehenden Konsensus sowie als Grundlage für die wahre Roma- Identität ansehen. Nehmen wir an, dass die soziale Frustration, die sich bei den Roma in sozialen Bereichen wiederspiegelt, ihren Ursprung im vorherigen Schulsystem findet, welcher (mit der Absicht der sofortigen Assimilation) für den Roma-Schüler große Barrieren geschaffen hat. Diese blockierten die Ausnutzung seiner tatsächlichen Entwicklungspotentiale.

Schüler, die über einen längeren Zeitraum schulische Mißerfolge erfahren haben, vermittelte die Schule das Gefühl der Ohnmacht, der gescheiterten Existenz, welche relativ schnell als asozial angesehen wird. Das Fehlen dialoger Formen sowie unzureichende Möglichkeiten zur Entwicklung eigener Roma- Kultur in Schulen, sind der Grund dafür, dass heutzutage die Entwicklung der Roma- Identität angestrebt wird, in der Annahme, dieses entspricht der sozialen Intergration. Dies sind auch Gründe dafür, wieso ich mich in meiner Arbeit der Erziehung und Bildung von Roma- Schülern gewidmet habe. Imaginäre Situation, Darstellung fiktiver Situationen, Suchen entsprechender Methoden beim Lehren konkreter Fächer dies alles sind Mittel, mit deren Hilfe die Fiktion zu einer Anleitung zum Lösen bestimmter Lebenssituationen werden könnte.

\section{Einige Beispiele zur Ausnutzung fiktiver Situationen in den Lernfächern Geschichte und Sozialkunde}

Ich stelle hier einige Spiele vor, die nicht nur als Mittel zum Aneignen neuer Informationen genutzt werden können, sondern auch als eine Methode, mit der man sich ethische Werte, die nötig sind beim Umgang mit anderen Mitmenschen, aneignet. Diese Spiele, durchgeführt zur Lösung fiktiver Situationen, haben es zum Ziel, die Identität der Roma- Kinder in der Schule sowie ihr Selbstwert- 
gefühl, was die Zugehörigkeit zu der Roma- Familie (romipen) anbetrifft, zu steigern. Es ist das Gefühl einer tiefen Verankerung in der Roma- Kultur und das emotionale, ethische und philosophische Zusammenspiel zwischen Menschen mit Roma- Abstammung.

\section{Geschichte}

Museum

Kleinere Gruppen von Kindern (Arbeitsteams) bekommen als Aufgabe ein neuartiges Museum einzurichten. Es sollte sich dabei um ein Museum handeln, das es noch nicht gibt. An den Kindern liegt es, sich auszudenken, was in diesem Museum ausgestellt werden sollte und warum. Am Ende des Projekts sollte eine Diskussion statt finden.

Ethischer Sinn des Spiels: In Schulklassen mit Roma- Kindern sollte ein Plan zur Errichtung eines Roma- Kulturmuseums erstellt werden. In der Diskussion können die Kinder dann Gründe dafür finden, wieso dieses errichtet werden sollte. Das Spiel sollte die Kinder zum Nachdenken über das Bedürfnis eines Menschen, die eigene Kultur kennen zu lernen, animieren. Die Kinder müssen wissen, zu wen sie gehören und an was sie sich als Individuum orientieren können. Neben der Erziehung zur Identifikation fördert das Spiel auch die Toleranz gegenüber anderen Kulturen.

\section{Historische Spiele}

Erstellen fiktiver Situationen in verschiedenen Stationen der menschlichen Geschichte:

Ethischer Sinn der Spiele: Die Kinder erhalten nicht nur Informationen $\mathrm{zu}$ einem bestimmten Lebensabschnitt, sondern lernen auch die Probleme kennen, mit denen frühere Generationen zu kämpfen hatten. Die Spiele sind mit Absicht in Form eines Dramas 
konzipiert und mit stimulierenden Elementen versehen, damit sie auch Spaß und Entspannung bringen.

Historisches Spiel kann für die Roma- Kinder zum Ausgangspunkt werden, um die geschichtliche Situation der Roma besser nachempfinden zu können (Nachdenken über die Abstammung, Kennenlernen der Kultur ihrer Urheimat - Indien, der Lebensweise, Wohngewohnheiten, Kleidungsstils etc.). Inspirationen dazu kann man zum Beispiel in dem Buch von Emilia Horvath: Zigeuner in der Slowakei (Geschichtlich- ethnographischer Umriss, Bratyslava 1964) finden. Eine weitere Quelle stellen u. A. die Märchen der Schriftstellerin Elena Lackowa dar sowie die Werke von Vlado Olah und anderen Roma- Schriftstellern.

\section{Der Zauberdiamant}

Wahrscheinlich hat jedes Volk oder frühere Volksstämme im Laufe der Geschichte Situation der Gefangenschaft, der Unterdrückung erfahren, aus der es versuchte auszubrechen. Dazu reichte aber nicht die Anstrengung einzelner Menschen oder einiger Helden. Nötig waren hier unzählige Bemühungen, Vereinigung aller möglicher Kräfte. Nur dies führte zu dem großartigsten aller Gefühle, zum Freiheitsgefühl, sowohl beim Individuum als auch beim ganzen Volk. Einen besonderen Befreiungsweg durchleben Gemeinschaften, die in Diaspora leben und keine eigene, alle Menschen vereinigende Heimat besitzen. Zu derartigen Gruppen gehören auch die Roma. Wenn wir den Geschichtsunterricht so gestalten wollen, dass alle Schüler den Befreiungsprozess miterleben und damit wir bezeugen können, dass sie die Rolle der vergangenen Generation übernehmen und verstehen, können wir folgendes Spiel (das starke Emotionen hervorruft) zum Einsatz bringen. Autorin dieses Spiels ist S. Pavelková (Machková, 1992).

Der Spielleiter, - ein guter Sprecher - erzählt folgende fiktive Geschichte: 
In einem Land lebten gutherzige Menschen, die niemanden je Leid zugefügt haben. In ihre Nachbarschaft zogen Feinde ein, die eifersüchtig auf ihr Glück, ihre Güte und ihre Fähigkeit zu lieben waren. Deswegen haben die Feinde sie des Sehens und Sprechens beraubt. Die Menschen in diesem Land konnten sich fortan nur durch Töne artikulieren oder - bei persönlichen Kontakt - durch Berührungen. Eines Tages kam der gute Zauberer zu ihnen i versprach, dass sie ihr Augenlicht und die Sprache zurückerlangen, wenn sie nur den Diamant finden, der irgendwo in den Auen verloren ging. Es reicht aus, dass man diesen Diamant an die Stirn legt und der Mensch wird wieder sehen und sprechen können. Alle Mitspieler haben geschlossene Augen und können sich frei bewegen. Das Spiel beginnt mit dem Zusammenrufen der Spielteilnehmer. Es bilden sich kleinere Gruppen, die sich wiederum mit anderen Gruppen verbinden, bis eine Reihe oder ein Kreis aus allen Spielern entsteht. Danach fangen alle an, den Diamant zu suchen. Auf den Knien suchen sie den Boden ab. Der Spielleiter legt einen Ersatzgegenstand - z. B. einen Stein - auf den Boden, aber erst während des Spiels. Am besten, wenn er diesen Stein demjenigen Spieler übergibt, bei dem er eine „ehrliche“ Vorgehensweise vermutet. Für die Suche sollte genügend Zeit zur Verfügung stehen, damit die nötige Spannung aufgebaut werden kann und es seitens der Spieler genügend Motivation gibt, den Diamant wirklich finden zu wollen.

Jeder Spieler, der den Diamant findet, legt ihn an die Stirn und erlangt dadurch das Augenlicht wieder. Also macht er die Augen auf mit derartigen Gefühl, als ob er zum ersten mal in seinem Leben etwas sehen würde. Gleichzeitig darf er aber die anderen nicht vergessen, darf den Stein nicht für sich behalten, sondern sollte ihn an andere Mitspieler weitergeben, damit sie eine ähnliche Katharsis (seelische Befreiung) erleben dürfen.

Bei guter Spielführung dauert das Spiel verhältnismäßig lang: eine oder sogar zwei Stunden. Nach dem Spielschluss sollte man kein neues anfangen, sondern eine Diskussion aufnehmen über den Verlauf des Spiels, da die emotionalen Erlebnisse sehr stark sind. 
Ethischer Sinn des Spiels: Dieses Spiel symbolisiert den Weg zur Freiheit und ermöglicht es, in fiktiven Model- Bedingungen die Katharsis (seelische Befreiung) zu erlangen. Beim genauen Kennenlernen der eigenen Geschichte, können die Spielteilnehmer deren einzelne Zeitabschnitte durchleben. Sie sind der Weg zu einem würdigen und freien Leben, zur Suche nach der wahren Heimat, wo die gegenseitige Solidarität unter der Familien und Klans sowie Erfahrungen aus dem hundertjährigen Migrationprozess das Leben der Roma nicht gerade einfach gestalteten.

Ich nehme an, dass man seine gegenwärtige Situation, nicht ohne den "Einsatz" der Situationen aus der Vergangenheit verstehen kann, ohne dass man sich der moralischen Bedeutung der damaligen Zeit bewusst wird. Weil die Mehrheitsgesellschaft den Roma immer schon missgünstig gegenüberstand, weil sie ihre Anwesenheit nah bei sich nicht ertragen konnte.

"Lass die Hunde los, die Zigeuner sind da" - das war die Parole des viktorianischen Englands gegen das Roma- Volk. Dagegen hat der deutsche Rassismus versucht, Roma, als eine Rassen- Untergruppe völlig auszurotten. In der Zeit der Konzentrationslager, aus denen es fast nie ein Zurückkommen gab, hatte das Suchen nach dem "Zauber- Diamant" eine ganz wichtige Funktion gespielt. Das Spiel erfüllte hier seinen Sinn - es ging um Durchleben des Befreiungswegs eigener Vorfahren. Auch wenn die Situation für die Roma- Häftlinge in Auschwitz nahezu aussichtslos war. Wie die Roma in ihren Liedern, mit den Titel „Aušvicate hi kher báro“ (In Auschwitz ist ein großes Gefängnis) über das Auschwitz- Leid klagen.

\section{Gesellschaftslehre}

\section{Mond- Staat}

Auf dem Mond ist eine Gemeinschaft entstanden, die aus Menschen verschiedener Kulturen besteht. Die Kinder sollen überlegen, wie sie diese Gemeinschaft organisieren können, welche Rechte 
man ihr zuspricht, welche Führungsart einsetzt, damit es zu keinen Konflikten kommt und damit Probleme durch Versöhnung in Rahmen einer Diskussion gelöst werden.

Ethischer Sinn des Spiels: Bei den Roma- Kindern oder in gemischten Schulklassen kann man auf diese Art und Weise ethische Prinzipien der Kommunikation und des Dialogs zwischen Angehörigen verschiedener Völker durchsetzen. Konkret handelt es sich hierbei um interkulturelle Beziehungen und um das Erziehen zur Diskussion. Bei diesem Spiel oder anderen Spielen ähnlichen Charakters können sich die Kinder auch die Methoden der Versöhnung durch Selbstbehauptung aneignen. (z. B. asertivní komunikace - Vališová, 1991, S. 56-63).

\section{Überlegungen zu den einzelnen Roma- Sprichwörtern}

Wichtig für Roma- Schüler als Ausgangspunkt zum Nachdenken und zur Dramatisierung von Roma- Sprichwörtern (weise Worte alter Roma).

Mit den Kindern lassen sich fiktive Situationen darstellen, deren Handlung auf folgenden Sprichwörtern basiert:

- ein hungriges Herz ist schlimmer als hungriger Bauch

- wer etwas verfolgt, der findet es

- das was für dich bestimmt ist, kann dir keiner nehmen; das was für das Schicksal parat hält, bleibt dir erhalten

- jeder Mensch ist anders, aber wir alle sind Menschen

- wenn du mich für einen Hund hältst, leine mich an, wenn ich für dich ein Mensch bin, bring mir Respekt entgegen.

Das Spiel zur Bestimmung der Roma- Identität: Wer bin ich?

Wir beginnen mit der Beschreibung einer fiktiven, imaginären Situation. Die Schüler entspannen sich, legen oder setzen sich bequem hin, schließen die Augen und hören sich eine fiktive Ge- 
schichte an: "Ihr geht durch den Wald, auf einer unebenen Straße. Es ist neblig, aber langsam lichtet sich der Nebel und ihr findet euch auf einer grünen Wiese wieder. Man kann Rauch in der Luft spüren. Ihr bemerkt eine Feuerstelle, das Feuer erlischt langsam. Rundherum liegen Steine und einige vergessene Sachen. Zwischen den Sachen leuchtet etwas - ein Taschenmesser. Wahrscheinlich wurde dieser von einem Jungen vergessen, der in deinem Alter sein konnte. Gleich daneben liegt ein rotes Tuch. Wahrscheinlich gehörte es dem Mädchen, welches dem aus unserer Klasse ähnlich sieht. Würde uns nicht soviel Zeit und Raum von diesen Kindern trennen, die das Lagerfeuer verlassen und mit der Familie weiter ziehen mussten, könnten wir sie hier bestimmt treffen. Und was wäre, wenn wir eine Geschichte über sie schreiben wurden, wie könnten sie sein?“.

Die Kinder fühlen sich jetzt in die Rolle der entfernten Verwandten hineinversetzt und überlegen, wie diese sein könnten. Danach schreibt jedes Kind seine Vorstellungen auf ein Blatt Papier. Zum Schluss lesen sich die Kinder die Notizen gegenseitig vor.

Vorschläge zum Aufbau der Geschichte:

- Wie sah der Junge, das Mädchen aus?

- Wie lebte er oder sie?

- Welche Träume hatte diese Person? Was wollte sie in ihrem Leben erreichen?

- Hatte sie irgendwelche Probleme?

- Kenne ich das Problem aus meinem eigenem Leben?

- Wenn ich dem Jungen/Mädchen einen Satz sagen könnte, wie würde dieser lauten?

Eine Abwandlung des Spiels: im Spiel geht es in erster Linie im die Zugehörigkeit zu der Roma- Familie. Die Kinder sollten sich aber auch in die Rolle der Nicht- Roma- Kinder hineinversetzen können.

Ethischer Sinn des Spiels: Das Spiel hilft dabei, dass sich der Roma- Schüler mit sich selbst identifiziert, sich innerlich einem Volk zugehörig fühlt (innerliche Identifikation). Indem er sich in die Rolle und die Situation der Nicht- Roma- Kinder versetzt, lernt der 
Roma- Schüler ein anderes Kind als einen gleichwertigen Partner und einen Vertreter der Mehrheitsgesellschaft zur respektieren (äußerliche Identifikation).

\section{Schlussfolgerung}

Der oben beschriebener Plan, in dem der Lehrer mit Hilfe von Erschaffung und Lösung fiktiver Situationen den Schüler schrittweise zum Interesse an einer bestimmten Ideologie, zum Kern der Sache führt, ist gleich zu setzen mit der Erziehung eines RomaKindes zu romipen. Art und Weise, wie romipen von der Pädagogin Lýdia Lehoczká am Institut für Roma- Studien der Universität Konstantyna-Filozofa in Nitra, in ihrer Veröffentlichung Kultúrna identita Rómov (Kulturelle Identität der Roma) dargestellt wird, führt zur Erklärung der Philosophie des Denkens bei Roma. Im romipen finden sich die wichtigsten Grundsätze für die Roma- Situation in der Welt. Und $\mathrm{zu}$ diesem romipen sollte auch ein RomaSchüler hingeführt werden, nicht nur in der eigenen Familie, sondern auch in der Schule.

Andere anregende Methoden, die man auch im Unterricht einsetzen kann, sind die oben aufgeführten Spiele. Romipen, wie L. Lehoczká es in ihrer Arbeit beschreibt: „bildet die Basis der kulturellen Roma- Identität. Romipen ist die Form persönlicher Identität und Gruppen- Identität, das Gefühl der Zugehörigkeit zu eigenem Volk. Romipen bildet die Summe kultureller Werte, sowohl im geistigen als auch materiellen Bereich. Romipen bedeutet persönliche Identifizierung mit der Roma- Kultur, mit den familiären Traditionen, mit unseren Wurzeln und der Abstammung" (Lehoczká, 2006, S. 57). Zu den wichtigsten Werten des romipen, die durch zwischenmenschliche Beziehungen ausgedrückt werden, zählt der Respekt. „Respekt stellt im Sinne der romipen eine wirklich wertvolle und tiefe Beziehung dar. Er sollte im menschlichen Leben eine besondere Stellung einnehmen. Er wird als eine Art des Gebens aus 
unseren Innerem angesehen. Somit ist es auch ein Apel an die Roma, dass diese lernen, das Leben um sich herum zu begreifen... Leben in Reinheit der Gedanken und des Herzens. Respekt schafft auch Raum für Weisheit" (Lehoczká, 2006, S. 60).

Dieser ist auch ein richtiger Platz zur philosophischen Überwindung der Welt, die uns umgibt, sowie der Welt in unserem Innernsten. Die Schule sollte auch in dieser Richtung wirken, bei der Erziehung des Schülers zum Verständnis des Lebens, bei seinem Bestreben nach Lebensweisheit, - für den Roma- Schüler entsprechend - romipen. Erstellung und Realisierung vorgegebener, fiktiver Situations- Methoden beim Unterrichten der Roma- Schüler ist eine von vielen Mitteln, welche die Identität sowie die Autonomie der Roma- Kinder im schulischen Leben stärken.

\section{Literatur}

HLAVSA J., LANGOVÁ M., VŠETEČKA J., Člověk v životnich situacích, Akademia, Praha 1987.

LEHOCZKÁ L., Kulturná identita Rómov (teoreticko-empirická analýza vybraných okruhovä), Univerzita Konštantína Filozofa v Nitre, Fakulta sociálnych vied a zdravotníctva, Nitra 2006.

MACHKOVÁ E., Metodika dramatické výchovy, Artama, Praha 1992.

PEŠKOVÁ J., SCHÜCKOVÁ L., Já, člověk, SPN, Praha 1991.

VAlišovÁ A., Asertivita v rodině a ve škole, Praha 1991, s. 56-63.

\section{Rozwój uczniów romskich: możliwości wykorzystania metod sytuacyjnych w edukacji kulturowej}

Streszczenie

Ważnym środkiem w pedagogice, który ma służyć edukacji uczniów, są metody sytuacyjne. Nauczyciel może w swojej pracy zastosować te metody, w których wykorzystuje się fikcyjne sytuacje jako środek do przygotowania uczniów do real- 
nego życia. Odgrywanie różnych ról daje możliwość doświadczanie różnych sytuacji, które mogą pojawić się życiu. Ta okoliczność, wykorzystanie metod sytuacyjnych, powinna być brana pod uwagę w edukacji uczniów romskich w rozwoju i utrzymaniu tożsamości Romów.

SŁOWA KLUCZE: edukacja, kultura romska, metody sytuacyjne 